\title{
Precipitation kinetics of $\mathrm{Al}_{3} \mathrm{Zr}$ and $\mathrm{Al}_{3} \mathrm{Sc}$ in aluminum alloys modeled with cluster dynamics
}

\author{
Emmanuel Clouet ${ }^{a, *}$, Alain Barbu ${ }^{a}$, Ludovic Laé ${ }^{b}$, \\ Georges Martin ${ }^{\mathrm{c}}$ \\ a Service de Recherches de Métallurgie Physique, CEA/Saclay, \\ 91191 Gif-sur-Yvette, France \\ ${ }^{\mathrm{b}} L T P C M / E N S E E G, I N P G$, B.P. 75 , \\ 38402 Saint Martin d'Hères, France \\ ${ }^{\mathrm{c}}$ Cabinet du Haut-Commissaire, CEA/Siège, \\ 31-33, rue de la Fédération, \\ 75752 Paris cedex 15, France
}

\begin{abstract}
Precipitation kinetics of $\mathrm{Al}_{3} \mathrm{Zr}$ and $\mathrm{Al}_{3} \mathrm{Sc}$ in aluminum supersaturated solid solutions is studied using cluster dynamics, a mesoscopic modeling technique which describes the various stages of homogeneous precipitation by a single set of rate equations. The only parameters needed are the interface free energy and the diffusion coefficient which are deduced from an atomic model previously developed to study the same alloys. A comparison with kinetic Monte Carlo simulations based on the vacancy diffusion mechanism shows that cluster dynamics correctly predicts the precipitation kinetics provided a size dependent interface free energy is used. It also manages to reproduce reasonably well existing experimental data.
\end{abstract}

Key words: precipitation, kinetics, aluminum alloys, cluster dynamics PACS: 64.60.Cn, 64.60.-i, 64.70.Kb, 64.75.+g

\section{Introduction}

Transition elements are added to aluminum alloys so as to obtain small ordered precipitates and by this way decrease the alloy sensitivity to recrystallization.

* emmanuel.clouet@cea.fr

Preprint submitted to Elsevier Science

14 November 2018 
Among these elements, zirconium and scandium are the most efficient ones. They lead to the precipitation of respectively $\mathrm{Al}_{3} \mathrm{Zr}$ and $\mathrm{Al}_{3} \mathrm{Sc}$ with the $\mathrm{L}_{2}$ structure [1-6]. As recrystallization is controlled by the precipitate density and size, it is important to model precipitation kinetics in Al-Zr and Al-Sc alloys, in order to optimize the heat treatment and better control the alloy final state.

In a previous study $[7,8]$, one of us built an atomic model for the above two binary alloys and studied precipitation kinetics using Monte Carlo simulations based on the vacancy diffusion mechanism. The drawback of such an approach is that only short annealing times can be simulated which implies using high supersaturations. We showed that the classical nucleation theory manages to reproduce atomic simulations giving thus good confidence in the ability of mesoscopic models to quantitatively predict the nucleation stage of precipitation kinetics. In the present work, starting from the same atomic model, we build a mesoscopic modeling, the cluster dynamics [9-11] which is based on a set of rate equations describing in the same framework the three different stages of precipitation, i.e. nucleation, growth and coarsening. It requires only a limited number of parameters, the interface free energy and the solute diffusion coefficients, which can be quite easily deduced from atomic parameters. The range of supersaturations and annealing times that can be simulated are thus extended allowing a comparison with experimental data. Cluster dynamics technique has already been used to model precipitation kinetics in $\mathrm{Al}-\mathrm{Zr}$ and Al-Sc alloys in the spirit of nucleation theory [12]. The way we use cluster dynamics in the present paper is distinct, as will be shown below, and is based on the description of the alloy as a gas of clusters. Unlike the nucleation theory, our approach leaves no arbitrary choice for the parameters used.

In the first part of this article, cluster dynamics modeling is presented. We show then how precipitation kinetics obtained with this method compare with kinetic Monte Carlo simulations for both Al-Zr and Al-Sc systems and try to improve the kinetic description so as to obtain a better agreement. Finally, available experimental data are compared with simulations.

\section{Cluster dynamics}

In its strict sense, cluster dynamics rests on the description of the alloy undergoing phase separation as a gas of solute clusters which exchange solute atoms by single atom diffusion [9-11]. Clusters are assumed to be spherical and are described by a single parameter, their size or the number $n_{\mathrm{X}}$ of solute atoms $\mathrm{X}$ they contain. In such a description, there is no precisely defined distinction between the solid solution on the one hand and the precipitates on the

other hand, at variance with the classical nucleation theory: the distribution 
of cluster sizes is the only quantity of interest (for a detailed discussion see Ref. 13). In an undersaturated solid solution of nominal concentration $x_{\mathrm{X}}^{0}$ at equilibrium, the cluster size distribution is given by (cf. appendix A)

$$
\bar{C}_{n_{\mathrm{X}}}\left(x_{\mathrm{X}}^{0}\right)=\exp \left[-\left(G_{n_{\mathrm{X}}}-2 n_{\mathrm{X}} \mu\left(x_{\mathrm{X}}^{0}\right)\right) / k T\right]
$$

$G_{n_{\mathrm{X}}}$ being the free energy of a cluster containing $n_{\mathrm{X}}$ atoms and $\mu\left(x_{\mathrm{X}}^{0}\right)=$ $\left(\mu_{\mathrm{X}}\left(x_{\mathrm{X}}^{0}\right)-\mu_{\mathrm{Al}}\left(x_{\mathrm{X}}^{0}\right)\right) / 2$ the effective chemical potential in the solid solution, i.e. half the difference between the chemical potentials of the solute and the solvent atoms. In a supersaturated system, the cluster size distribution evolves in time as discussed below.

\subsection{Master equation}

The cluster dynamics technique describes the precipitation kinetics thanks to a master equation giving the time evolution of the cluster size distribution [9-11]. When only monomers can migrate, which is the case for Al-Zr as well as Al-Sc systems [7], the probability $C_{n_{\mathrm{X}}}$ to observe a cluster containing $n_{\mathrm{X}}$ solute atoms obeys the differential equations

$$
\begin{aligned}
\frac{\mathrm{d} C_{n_{\mathrm{X}}}}{\mathrm{d} t} & =J_{n_{\mathrm{X}}-1 \rightarrow n_{\mathrm{X}}}-J_{n_{\mathrm{X}} \rightarrow n_{\mathrm{X}}+1}, \quad \forall n_{\mathrm{X}} \geq 2 \\
\frac{\mathrm{d} C_{1}}{\mathrm{~d} t} & =-2 J_{1 \rightarrow 2}-\sum_{n_{\mathrm{X}} \geq 2} J_{n_{\mathrm{X}} \rightarrow n_{\mathrm{X}}+1},
\end{aligned}
$$

where $J_{n_{\mathrm{X}} \rightarrow n_{\mathrm{X}}+1}$ is the cluster flux from the class of size $n_{\mathrm{X}}$ to the class $n_{\mathrm{X}}+1$. This flux can be written

$$
J_{n_{\mathrm{X}} \rightarrow n_{\mathrm{X}}+1}=\beta_{n_{\mathrm{X}}} C_{n_{\mathrm{X}}}-\alpha_{n_{\mathrm{X}}+1} C_{n_{\mathrm{X}}+1}
$$

$\beta_{n_{\mathrm{X}}}$ being the probability per unit time for one solute atom to impinge on a cluster of size $n_{\mathrm{X}}$ and $\alpha_{n_{\mathrm{X}}}$ for one atom to leave a cluster of size $n_{\mathrm{X}}$.

In order to handle large cluster sizes, the set of differential equations 2 and 3 are integrated by considering cluster sizes in a discrete way up to a maximal size $\left(n_{\mathrm{X}} \sim 100\right)$ and by using a continuous variable with an increasing step beyond [14].

\subsection{Condensation rate}

When the solute long-range diffusion controls the precipitation kinetics, the condensation rate is obtained by solving the diffusion problem in the solid 
solution around a spherical precipitate (cf. appendix B). For a cluster of radius $r_{n_{\mathrm{X}}}$, this condensation rate takes the form $[15,16]$

$$
\beta_{n_{\mathrm{X}}}=4 \pi r_{n_{\mathrm{X}}} \frac{D_{\mathrm{X}}}{\Omega} C_{1}
$$

where $D_{\mathrm{X}}$ is the diffusion coefficient of the solute at infinite dilution of the solid solution and $\Omega$ is the mean atomic volume corresponding to one lattice site. We checked by studying growth of planar precipitates that precipitation kinetics can truly be assumed to be controlled by solute long range diffusion and that the diffusion coefficients deduced from kinetic Monte Carlo simulations are in good agreement with the experimental ones entering equation 4 [17].

\subsection{Evaporation rate}

By contrast with the condensation rate, the evaporation rate $\alpha_{n_{\mathrm{X}}}$ cannot be obtained directly. It has to be deduced from $\beta_{n_{\mathrm{X}}}$ using the equilibrium cluster size distribution (Eq. 1).

The evaporation rate is obtained assuming that it is an intrinsic property of the cluster and therefore does not depend on the solid solution surrounding the cluster ${ }^{1}$. This means that the cluster has enough time to explore all its configurations between the arrival and the departure of a solute atom. Thus $\alpha_{n_{\mathrm{X}}}$ should not depend on the nominal concentration of the solid solution and can be obtained by considering any undersaturated solid solution of nominal concentration $x_{\mathrm{X}}^{0}$. Such a solid solution is stable. Then there should be no energy dissipation. This involves that all fluxes $J_{n_{\mathrm{X}} \rightarrow n_{\mathrm{X}}+1}$ equal zero. Using equation 3 , one obtains

$$
\begin{aligned}
\alpha_{n_{\mathrm{X}}+1} & =\bar{\alpha}_{n_{\mathrm{X}}+1}\left(x_{\mathrm{X}}^{0}\right) \\
& =\bar{\beta}_{n_{\mathrm{X}}}\left(x_{\mathrm{X}}^{0}\right) \frac{\bar{C}_{n_{\mathrm{X}}}\left(x_{\mathrm{X}}^{0}\right)}{\bar{C}_{n_{\mathrm{X}}+1}\left(x_{\mathrm{X}}^{0}\right)},
\end{aligned}
$$

where overlined quantities are evaluated in the solid solution at equilibrium. In particular, the equilibrium cluster size distribution is given by equation 1 . This finally leads to the following expression of the evaporation rate

$$
\alpha_{n_{\mathrm{X}}+1}=4 \pi r_{n_{\mathrm{X}}} \frac{D_{\mathrm{X}}}{\Omega} \exp \left[\left(G_{n_{\mathrm{X}}+1}-G_{n_{\mathrm{X}}}-G_{1}\right) / k T\right] .
$$

\footnotetext{
1 Sometimes, one assumes instead that a constraint equilibrium exists for the clusters in the supersaturated solid solution and that the expression 1 holds even for concentrations higher than the solubility limit $x_{\mathrm{X}}^{e q}$. Katz and Wiedersich [18] pointed that this assumption can lead to the same expression of the evaporation rate as the one we used. In particular, this is true when the condensation rate varies linearly with the monomer concentration which is the case here.
} 
As the condensation rate varies linearly with the monomer concentration, the contribution of the effective chemical potential cancels out in the expression 5 of $\alpha_{n_{\mathrm{X}}}$ : we recover our starting assumption. Indeed, all informations concerning the solid solution are contained in this chemical potential. Therefore $\alpha_{n_{\mathrm{X}}}$ only depends on the clusters free energies and not on the overall state of the gas of clusters. Especially, the evaporation rate is independent of the nucleation free energy, i.e. the free energy gained when nucleating a precipitate out of the solid solution: such concepts are useful in the classical nucleation theory but play no role in cluster dynamics. This can be seen by dividing the cluster formation free energy into a volume and an interface contributions [7],

$$
G_{n_{\mathrm{X}}}-2 n_{\mathrm{X}} \mu\left(x_{\mathrm{X}}^{0}\right)=4 n_{\mathrm{X}} \Delta G^{n u c}\left(x_{\mathrm{X}}^{0}\right)+\left(36 \pi n_{\mathrm{X}}^{2}\right)^{1 / 3} a^{2} \sigma_{n_{\mathrm{X}}},
$$

$\Delta G^{n u c}\left(x_{\mathrm{X}}^{0}\right)$ being the nucleation free energy of a solid solution of nominal concentration $x_{\mathrm{X}}^{0}, \sigma_{n_{\mathrm{X}}}$ the interface free energy of a cluster containing $n_{\mathrm{X}}$ solute atoms and $a$ the lattice parameter. This leads to the following expression of the evaporation rate

$$
\begin{aligned}
\alpha_{n_{\mathrm{X}}+1}= & 4 \pi r_{n_{\mathrm{X}}} \frac{D_{\mathrm{X}}}{\Omega} \\
& \exp \left[(36 \pi)^{1 / 3} a^{2}\left(\left(n_{\mathrm{X}}+1\right)^{2 / 3} \sigma_{n_{\mathrm{X}}+1}-n_{\mathrm{X}}{ }^{2 / 3} \sigma_{n_{\mathrm{X}}}-\sigma_{1}\right) / k T\right] .
\end{aligned}
$$

Looking at the expression 4 of the condensation rate and the expression 8 of the evaporation rate, one sees that the only parameters needed by cluster dynamics are the diffusion coefficient and the interface free energy. There is no need to know the nucleation free energy ${ }^{2}$ in opposition to other mesoscopic models based on classical nucleation theory following Langer and Schwartz approach [19] as modified by Kampmann and Wagner [1, 20-22]. In cluster dynamics, thermodynamics of the solid solution is described thanks to a lattice gas model and therefore the nucleation free energy results from this description and is not an input of the modeling.

\subsection{Definition of precipitates}

The master equation which describes cluster dynamics yields the full cluster distribution as a function of time. In order to follow the precipitation kinetics and make the link with experimental results, it is convenient to define mean values such as the precipitates mean size or their density so as to characterize

2 Some cluster dynamics studies [12] makes the evaporation rate depend on the nucleation free energy because a constrained equilibrium of the cluster size distribution has been considered so as to reproduce the instantaneous monomer concentration $C_{1}$ and not the total solid solution concentration $x_{\mathrm{X}}^{0}$ as it should be. 
kinetics by the evolution of these quantities. Therefore, the solid solution has to be discriminated from the precipitates: one needs to define an arbitrary threshold size $n_{\mathrm{X}}^{*}$ above which clusters represent stable precipitates and below which they represent fluctuations in the solid solution. One natural choice for this threshold size is the initial critical size given by the classical nucleation theory,

$$
n_{\mathrm{X}}^{*}=-\frac{\pi}{6}\left(\frac{a^{2} \sigma_{n_{\mathrm{X}}^{*}}}{\Delta G^{n u c}\left(x_{\mathrm{X}}^{0}\right)}\right) .
$$

This requires to calculate for a given temperature and a given composition of the solid solution the nucleation free energy. This cannot be directly done with the lattice gas model used by cluster dynamics as the effective chemical potential $\mu_{\mathrm{X}}\left(x_{\mathrm{X}}^{0}\right)$ appearing in equation 7 and needed to obtain $\Delta G^{n u c}\left(x_{\mathrm{X}}^{0}\right)$ is implicit. This potential can be deduced from the simulation when a stationary cluster size distribution is observed ${ }^{3}$ but it is not known a priori. Nevertheless, the nucleation free energy can be estimated by other means and in particular thanks to different mean field approximations. In reference 7 it was shown that the cluster variation method (CVM) $[23,24]$ in the tetrahedron-octahedron approximation leads to a good prediction of $\Delta G^{n u c}\left(x_{\mathrm{X}}^{0}\right)$. Therefore the value of the threshold size is chosen equal to that of the critical size in the classical nucleation theory when CVM is used to calculate the nucleation free energy. Thermodynamics, and then the nucleation free energy, deduced from CVM can differ from the ones really corresponding to cluster dynamics. Nevertheless, this nucleation free energy is only used to calculate a threshold size and as the aim of this paper is to compare precipitation kinetics obtained with Monte Carlo and cluster dynamics simulations, this is not a real issue. The key point is to use the same threshold size when comparing the two modeling techniques.

\section{Cluster dynamics simulations}

In references 7 and 8 one of us developed an atomic kinetic model for $\mathrm{Al}-\mathrm{Zr}$ and Al-Sc systems. Parameters were deduced from experimental data (Zr and Sc solubility limits in $\mathrm{Al}$ and diffusion coefficients) as well as from ab-initio calculations $\left(\mathrm{Al}_{3} \mathrm{Zr}\right.$ and $\mathrm{Al}_{3} \mathrm{Sc}$ free energy of formation). So as to compare the precipitation kinetics obtained from cluster dynamics with kinetic Monte Carlo simulations we have to deduce from this atomic model the interface free energies between the precipitates and the aluminum matrix. As for diffusion coefficients, we use in the cluster dynamics simulations the experimental values known for Zr and Sc impurity diffusion in aluminum [25-27]:

3 The effective chemical potential is then linked to the instantaneous monomer concentration by the relation $\mu=\left[G_{1}+k T \ln \left(C_{1}\right)\right] / 2$. 


$$
\begin{aligned}
& D_{\mathrm{Zr}^{*}}=728 \times 10^{-4} \exp (-2.51 \mathrm{eV} / k T) \mathrm{m}^{2} \cdot \mathrm{s}^{-1} \\
& D_{\mathrm{Sc}^{*}}=5.31 \times 10^{-4} \exp (-1.79 \mathrm{eV} / k T) \mathrm{m}^{2} \cdot \mathrm{s}^{-1} .
\end{aligned}
$$

As shown in Ref. 7,8, the diffusion model used in kinetic Monte Carlo does reproduce the above values.

\subsection{Interface free energy}

The interface free energy can be deduced from the atomic model by computing the free energies corresponding to planar interfaces in the three most dense packing orientations $\{111\},\{110\}$ and $\{100\}$ within the Bragg-Williams approximation and then by using the Wulff construction to obtain an isotropic average interface free energy $\bar{\sigma}[7]$. Such an interface free energy depends on the temperature for two reasons: atomic interactions used to obtain $\bar{\sigma}$ vary with temperature and there is a configurational entropy contribution due to the relaxation of the interface. On the other hand it does not depend on the size of the cluster and corresponds to the asymptotic limit of $\sigma_{n_{\mathrm{X}}}$. A direct calculation considering the partition functions of small clusters shows that $\sigma_{n_{\mathrm{X}}}$ slightly deviates from this asymptotic value at small sizes [7]. In the following, we test effects on cluster dynamics results due to this slight dependence of the interface free energy with the cluster size. Some authors $[9,11,28]$ already used an interface free energy depending on the cluster size so as to model copper precipitation in iron by cluster dynamics. In these studies, the size dependence was introduced to take into account the change with size of the precipitate structure. This is not the purpose here as precipitates keep their $\mathrm{L}_{2}$ structure whatever their size. We want to show how sensitive are cluster dynamics simulations to the interface free energy and why one needs to go beyond a model using a constant parameter.

In this purpose, for clusters containing no more than 9 solute atoms, the interface free energy is computed using Eq. 20 of Ref. 7. For clusters of size $n_{\mathrm{X}} \geq 10$, the interface free energy is obtained using an extension of the capillary approximation,

$$
\sigma_{n_{\mathrm{X}}}=\bar{\sigma}\left(1+c n_{\mathrm{X}}^{-1 / 3}+d n_{\mathrm{X}}^{-2 / 3}\right)
$$

where $c$ and $d$ respectively correspond to the line and point contributions [29]. The asymptotic value $\bar{\sigma}$ used is the one previously calculated thanks to the Bragg-Williams approximation and the Wulff construction [7] whereas coefficients $c$ and $d$ are obtained by a least square fit of the expression 20 of Ref. 7 for sizes $5 \geq n_{\mathrm{X}} \geq 9$. The curvature correction $c$ obtained with such a procedure is found to be negative in agreement with classical models [30,31]. Instead of using such an approximate procedure to obtain the interface free energy, this can be done more precisely by sampling thermodynamic averages 
with a Monte Carlo algorithm so as to compute the free energy difference between a cluster of size $n$ and one of size $n+1$ at a given temperature [29]. Interface free energies obtained by both methods are in good agreement [32].

As seen in figure 1, the difference between this interface free energy $\sigma_{n_{\mathrm{X}}}$ depending on the cluster size and its asymptotic limit $\bar{\sigma}$ is small. For instance, for the interface between $\mathrm{Al}_{3} \mathrm{Sc}$ and the solid solution at $T=500^{\circ} \mathrm{C}$, the maximal difference is obtained for sizes $n_{\mathrm{X}} \sim 10$ and is less than $5 \%$.

We run some cluster dynamics simulations with the interface free energy depending on the cluster size as given by Eq. 20 of Ref. 7 and Eq. 10 and some other simulations with a constant interface free energy taken equal to the asymptotic limit $\bar{\sigma}$. The comparison with kinetic Monte Carlo simulations (Fig. 2) shows that one obtains a better agreement when taking into account the size dependence: the number of precipitates as well as their mean size predicted by both simulation methods are then quite close. Although the size dependence of the interface free energy is small, it definitely improves the ability of cluster dynamics to well reproduce kinetic Monte Carlo results. This is true mainly for the precipitate density, the effect on precipitate mean size being less pronounced, especially when the supersaturation is low. The density is more sensitive than the mean size to the distribution of small clusters. As these clusters are the most affected by the variation with size of the interface energy, this explains why the greatest effect observed is for the precipitate density.

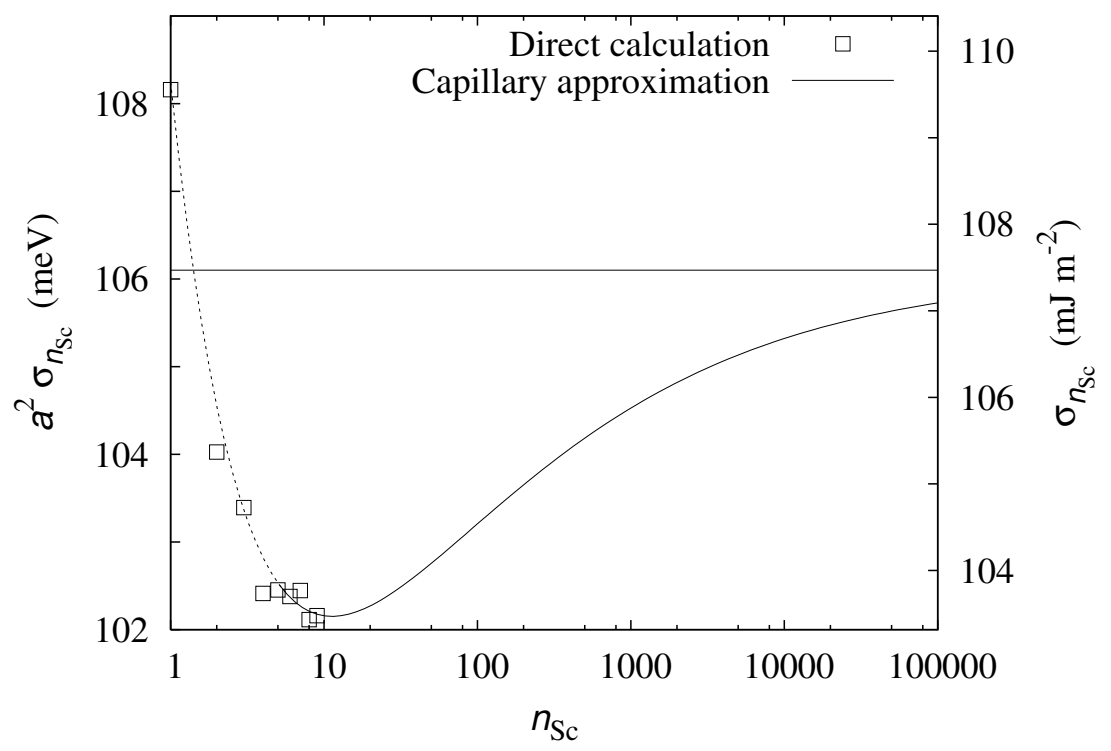

Fig. 1. Variation with the cluster size $n_{\mathrm{Sc}}$ of the interface free energy between the solid solution and $\mathrm{Al}_{3} \mathrm{Sc}$ at $T=500^{\circ} \mathrm{C}$. Symbols correspond to $\sigma_{n_{\mathrm{Sc}}}$ as given by the direct calculation of the cluster formation free energy (Eq. 20 of Ref. 7) and lines to the capillary approximation (Eq. 10) as well as to its asymptotic limit. 


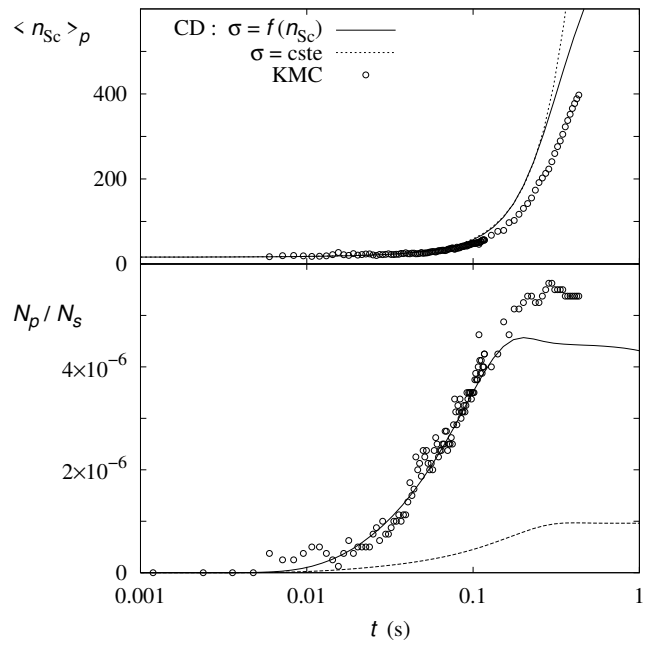

(a) $x_{\mathrm{Sc}}^{0}=0.4$ at. $\%\left(n_{\mathrm{Sc}}^{*}=16\right)$

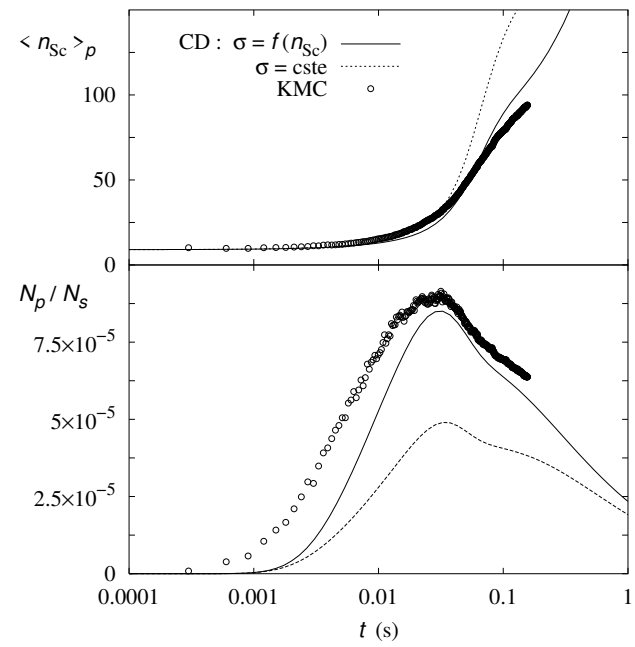

(b) $x_{\mathrm{Sc}}^{0}=0.75$ at. $\%\left(n_{\mathrm{Sc}}^{*}=9\right)$

Fig. 2. Precipitation kinetics of two supersaturated solid solutions of nominal concentration $x_{\mathrm{Sc}}^{0}=0.4$ at. $\%$ and $x_{\mathrm{Sc}}^{0}=0.75$ at. $\%$ at $T=500^{\circ} \mathrm{C}$ : evolution with time of the number $N_{p}$ of precipitates in the simulation box (normalized by the number of lattice sites $N_{s}$ ) and of their average size $\left\langle n_{\mathrm{Sc}}\right\rangle_{p}$. Full lines correspond to cluster dynamics simulations with an interface free energy depending on the cluster size (Eq. 20 of Ref. 7 and 10), dotted lines to simulations with a constant interface free energy and symbols to kinetic Monte Carlo simulations

In the following, all cluster dynamics simulations will use this size dependent interface free energy as input parameter.

\subsection{Precipitation kinetics}

Comparing, at a given temperature, the precipitation kinetics obtained with Monte Carlo and cluster dynamics simulations for different nominal concentrations of the solid solution (Fig. 3), one sees that cluster dynamics manages to reproduce the variations of the precipitate density as well as the variations of their mean size. For low supersaturations, the agreement is really good whereas for higher supersaturations there is a delay: cluster dynamics is too slow compared to kinetic Monte Carlo simulations by a factor of roughly 2 . Nevertheless, in all cases, the prediction of the precipitate maximal density at the transition between the growth and coarsening stages is correct. Cluster dynamics technique manages to reproduce the variations over three orders of magnitude of this maximal density in the range of concentrations considered in Al-Sc solutions at $T=450^{\circ} \mathrm{C}$ (Fig. 3). 
Fixing now the nominal concentration of the solid solution and varying the temperature, the comparison of results obtained with both simulation techniques leads to the same conclusions (Fig. 4). Cluster dynamics results still appear to be too slow at high supersaturations, but except this time delay, variations of precipitate density and of their mean size are well reproduced.
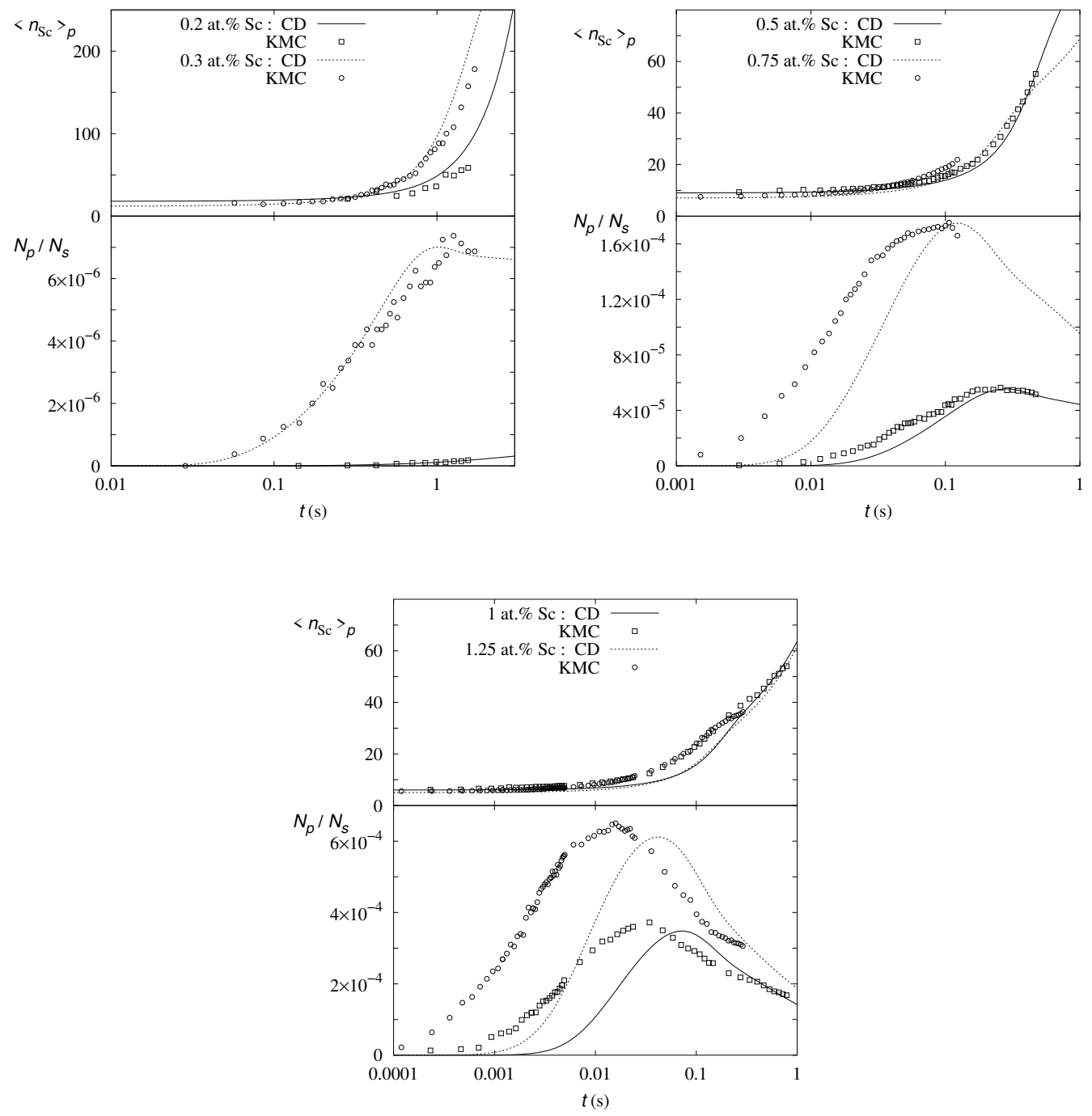

Fig. 3. Precipitation kinetics for different nominal concentrations of a supersaturated aluminum solid solution at $T=450^{\circ} \mathrm{C}$. Lines correspond to cluster dynamics simulations and symbols to kinetic Monte Carlo simulations. 


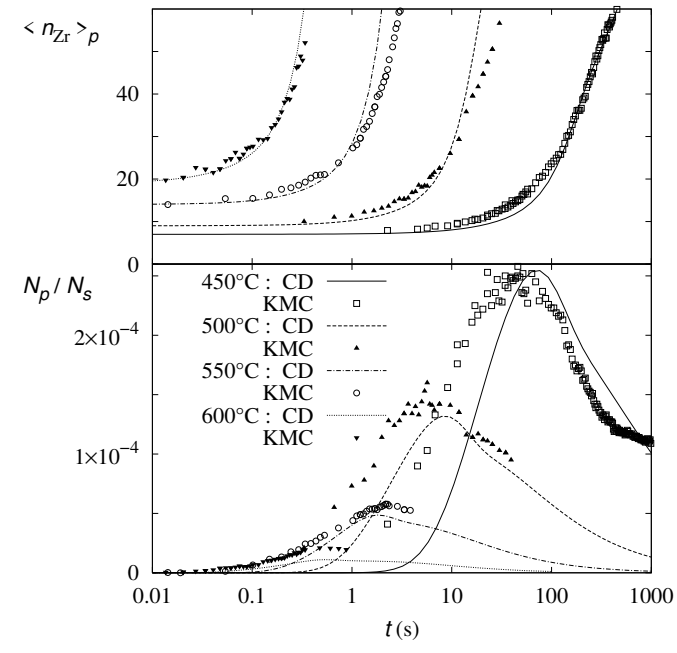

(a) $x_{\mathrm{Zr}}^{0}=1$ at. $\%$

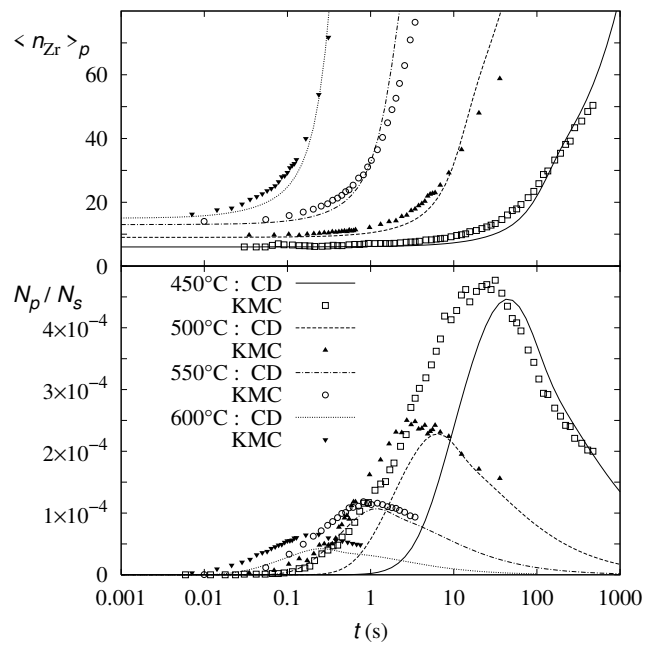

(b) $x_{\mathrm{Zr}}^{0}=1.25$ at. $\%$

Fig. 4. Precipitation kinetics for different temperatures of a supersaturated solid solution of nominal concentration $x_{\mathrm{Zr}}^{0}=1$. at. $\%$ and $x_{\mathrm{Zr}}^{0}=1.25$ at.\% 


\subsection{Cluster size distribution}

Cluster dynamics lead to a stationary cluster size distribution in the solid solution, i.e. for clusters smaller than a critical size $n_{\mathrm{X}}^{*}$ (Fig. 5), as predicted by classical nucleation theory. This stationary distribution corresponds to a constraint equilibrium existing for sub-critical clusters but nothing has been supposed concerning clusters bigger than the critical one. Using the capillary approximation to estimate the cluster formation free energy (Eq. 7), classical nucleation theory assumes this stationary distribution to be given by equation 1. Obviously, in this expression, the interface free energy has to be taken equal to the one used for cluster dynamics simulations and therefore we consider the size dependent interface free energy given by Eq. 20 of Ref. 7 and Eq.10. As for the nucleation free energy $\Delta G^{n u c}\left(x_{\mathrm{X}}^{0}\right)$, we already pointed that several thermodynamic approximations can be used to estimate it. One usually considers ideal or regular solid solution models to calculate $\Delta G^{\text {nuc }}\left(x_{\mathrm{X}}^{0}\right)$ but it was shown in reference 7 that at least CVM in the tetrahedron-octahedron approximation has to be used in order to obtain a good agreement between classical nucleation theory predictions and kinetic Monte Carlo simulations. The comparison of the stationary cluster size distributions in the solid solution obtained from cluster dynamics simulations with the ones predicted by classical nucleation theory leads to the same conclusion: one has to use CVM to calculate the nucleation free energy in order to get the right distribution

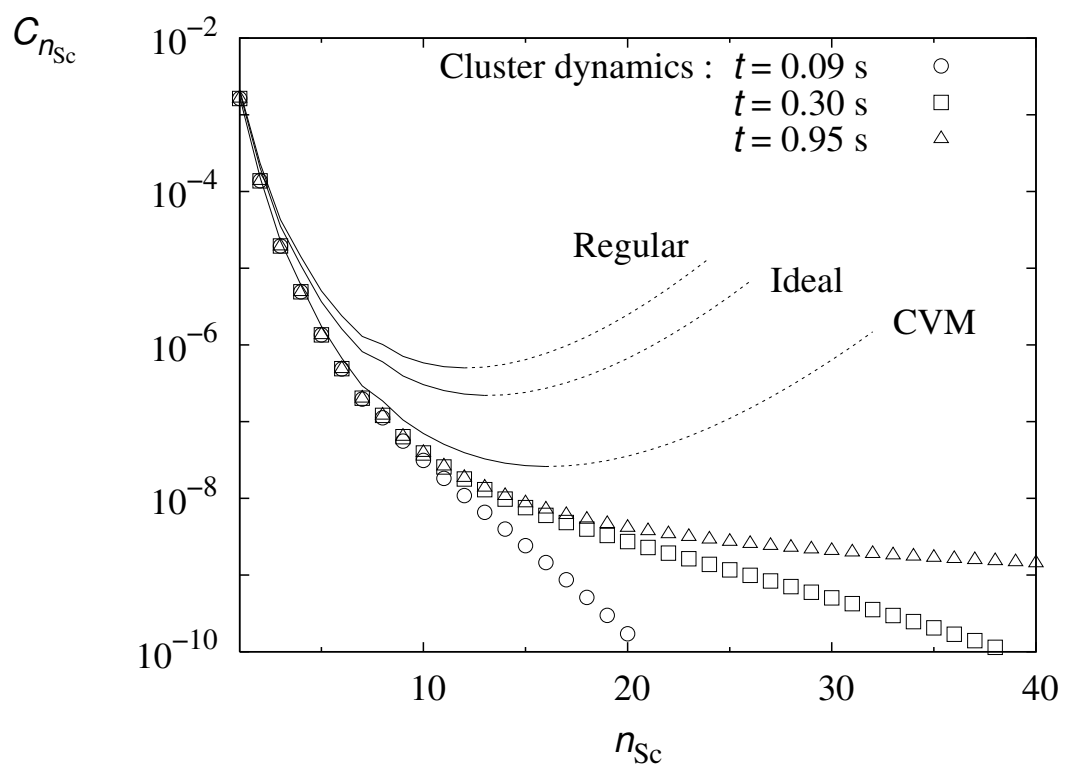

Fig. 5. Cluster size distribution of an aluminum solid solution of nominal concentration $x_{\mathrm{Sc}}^{0}=0.2$ at. $\%$ at $T=450^{\circ} \mathrm{C}$. Symbols correspond to cluster dynamics simulations and lines to prediction of classical nucleation theory with different mean-field approximations of the nucleation free energy. 
(Fig. 5) ${ }^{4}$. This result can appear quite surprising: although no information concerning the nucleation free energy has been used to run cluster dynamics simulations, this method leads to the stationary size distribution corresponding to the CVM. It seems that the lattice gas thermodynamic description used by the cluster dynamics manages to take into account short range order effects which are considered within CVM, in opposition to ideal and regular solid solution approximations.

\section{Effect of the overlap of diffusion fields}

As seen in the previous section, discrepancies between cluster dynamics and kinetic Monte Carlo simulations appear for high supersaturations, around 1 at.\% at $T=450^{\circ} \mathrm{C}$ for instance. Although the maximal precipitate density and its variations with temperature and supersaturation are correctly predicted, the precipitation kinetics as given by cluster dynamics are too slow. One possible origin of such a discrepancy might be in the expression for the condensation rate $\beta_{n_{\mathrm{X}}}$ : the later is computed solving, under stationary conditions, the diffusion equation around the cluster isolated in an infinite medium. This assumption looks reasonable for dilute solutions but its validity is not so clear for high supersaturations when clusters are quite close from each other: overlap of diffusion fields might alter the rate of solute impingement at clusters. Therefore, in order to see if a better agreement can be obtained between cluster dynamics and kinetic Monte Carlo simulations, it is worth trying to describe kinetic effects arising from interactions between the diffusion fields created around each precipitate and to incorporate those in cluster dynamics simulations.

In this purpose, we follow the approach proposed by Brailsford et al. [34] and already used by Smetniansky-De-Grande and Barbu in their modeling of the precipitation kinetics in $\mathrm{Fe}-\mathrm{Cu}$ alloys $[35,36]$. When calculating the monomer condensation rate on a given cluster, we assume that at distances greater than $r^{e x t}$, one half of the mean distance between precipitates, monomers feel influence of the cluster through its diffusion field as well as the one arising from other clusters through an effective medium characterized by a parameter $k$. The rate at which this effective medium absorbs monomers is given by $D_{\mathrm{X}} k^{2}\left(C_{1}-C_{1}^{e q}\right), C_{1}$ and $C_{1}^{e q}$ being respectively the instantaneous and the equilibrium monomer concentrations. This leads to the following expression of the condensation rate, depending on whether the radius $r_{n_{\mathrm{X}}}$ of the cluster

4 The difference between the distribution given by equation 1 and the observed stationary distribution during cluster dynamics simulations for size equal or slightly smaller than the critical one can be attributed to the Zeldovitch factor. Indeed, for the critical size, there should be a factor $1 / 2$ between the two distributions [33]. 
is smaller or greater than $r^{e x t}$ (cf. appendix B),

$$
\begin{array}{ll}
\beta_{n_{\mathrm{X}}}=4 \pi \frac{D_{\mathrm{X}}}{\Omega} r_{n_{\mathrm{X}}} C_{1} \frac{1+k r^{e x t}}{1+k\left(r^{e x t}-r_{n_{\mathrm{X}}}\right)}, & r_{n_{\mathrm{X}}} \leq r^{e x t}, \\
\beta_{n_{\mathrm{X}}}=4 \pi \frac{D_{\mathrm{X}}}{\Omega} r_{n_{\mathrm{X}}} C_{1}\left(1+k r_{n_{\mathrm{X}}}\right) \quad, & r_{n_{\mathrm{X}}} \geq r^{e x t} .
\end{array}
$$

Comparing this expression with the previous one (Eq. 4) where precipitates were assumed to be isolated, we see that the consideration of interactions between the different cluster diffusion fields through the introduction of this effective medium leads to a greater condensation rate.

So as to ensure that cluster dynamics simulations converge to equilibrium, i.e. a two-phases system consisting of $\mathrm{Al}_{3} \mathrm{X}$ precipitates embedded in a solid solution of composition $x_{\mathrm{X}}^{e q}$, evaporation rates need to be multiplied by the same factor as the condensation rates. They are now given by

$$
\begin{array}{ll}
\alpha_{n_{\mathrm{X}}}=\alpha_{n_{\mathrm{X}}}^{0} \frac{1+k r^{e x t}}{1+k\left(r^{e x t}-r_{n_{\mathrm{X}}-1}\right)}, & r_{n_{\mathrm{X}}-1} \leq r^{e x t}, \\
\alpha_{n_{\mathrm{X}}}=\alpha_{n_{\mathrm{X}}}^{0}\left(1+k r_{n_{\mathrm{X}}-1}\right) & r_{n_{\mathrm{X}}-1} \geq r^{e x t},
\end{array}
$$

where $\alpha_{n_{\mathrm{X}}}^{0}$ is the evaporation rate of an isolated cluster as given by equation 8. Through the parameters $k$ and $r^{e x t}$, the evaporation rates now depend on the solid solution considered.

As the effective medium we introduced has to be equivalent to the cluster assembly, the parameter $k$ is calculated so as to ensure that the quantity of monomers absorbed by this effective medium is equal to the quantity consumed by all clusters,

$$
D_{\mathrm{X}} k^{2}\left(C_{1}-C_{1}^{e q}\right)=2 J_{1 \rightarrow 2}+\sum_{n_{\mathrm{X}} \geq 2} J_{n_{\mathrm{X}} \rightarrow n_{\mathrm{X}}+1} .
$$

This equation has to be solved self-consistently at each step of cluster dynamics simulations. Starting from the value of $k$ corresponding to the previous iteration or from 0 for the first step, equations 11 and 12 allow to calculate the cluster condensation and evaporation rates and thus the different fluxes. Using equation 13 a new value of the parameter $k$ can be computed. To do so, the equilibrium monomer concentration $C_{1}^{e q}$ is required. It is simply given by the equilibrium cluster size distribution 1 when the nucleation free energy is null,

$$
C_{1}^{e q}=\exp \left(-(36 \pi)^{1 / 3} \sigma_{1} / k T\right)
$$

Precipitation kinetics obtained with cluster dynamics when considering interactions between the cluster diffusion fields are not really different from the ones previously obtained without such an interaction. At low supersaturations, no difference is observed whereas at higher supersaturations (Fig. 6) 
this interaction slightly speeds up the precipitation kinetics. Nevertheless, this does not really improve the agreement with kinetic Monte Carlo simulations and kinetics obtained with cluster dynamics still appear too slow. Therefore the discrepancies appearing at high supersaturations do not arise from the assumption of isolated clusters used to calculate the condensation rate but should have another origin. We checked too that the use of stationary conditions to compute the condensation rate is correct by using the whole solution, i.e. incorporating the transition state, of the diffusion equations [15] but this does not lead to a better agreement. Another possible origin of the discrepancy could be the use of a single parameter, their size, to describe clusters which involves considering clusters as spherical particles. This assumption might be too crude when computing the condensation rate but we did not manage to check this point as this spherical approximation is needed to solve the longrange diffusion equations.

As the correction taking into account the overlap of cluster diffusion fields does not really change cluster dynamics simulations, we will not consider it in the following section when comparing simulated kinetics with experimental

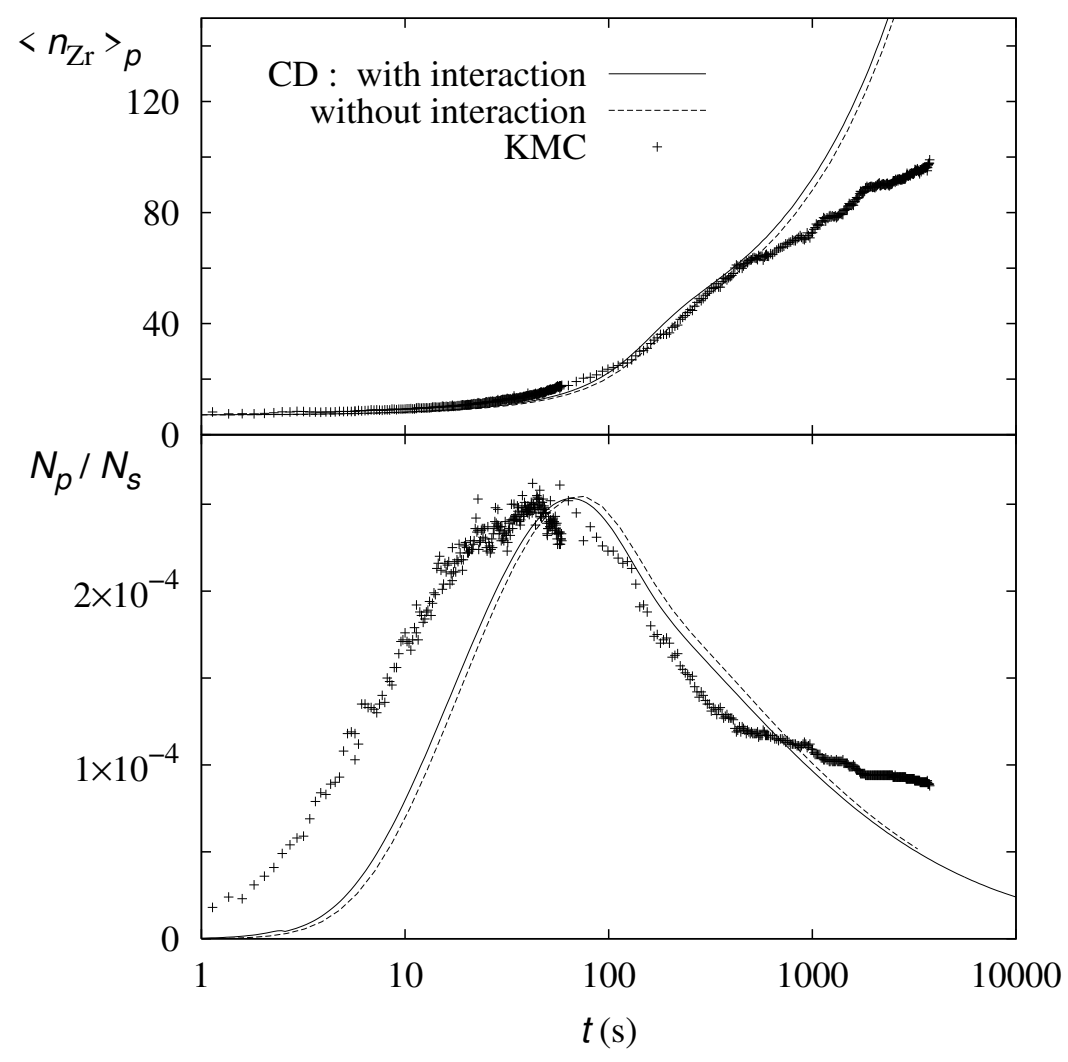

Fig. 6. Precipitation kinetics of a supersaturated solid solution of nominal concentration $x_{\mathrm{Zr}}^{0}=1$ at. $\%$ at $T=450^{\circ} \mathrm{C}$. Lines correspond to cluster dynamics simulations with or without interaction between the diffusion fields created around each cluster and symbols to kinetic Monte Carlo simulations. 
data: the condensation and evaporation rates will be those of isolated clusters as given by equations 4 and 8 .

\section{Comparison with experimental data}

Unlike kinetic Monte Carlo which can only simulate rapid processes (early stages of precipitation, high supersaturations), cluster dynamics can be used for low supersaturations and long annealing times. This allows a direct comparison with experimental studies. The precipitation kinetics in Al-Zr [1-3,37-40] as well as in Al-Sc system [4-6,22,41-46] has been studied by several groups. However, for the Al-Zr system, experimental data can hardly be directly compared to cluster dynamics simulations. Indeed, one observes two kinds of precipitates, some spherical ones and some rod-like shaped ones. Moreover, for low supersaturations, nucleation occurs heterogeneously and, Al-Zr being peritectic, high supersaturations are unreachable. Another difficulty arises from the fact that the $\mathrm{L}_{2}$ structure is unstable and that the stable $\mathrm{DO}_{23}$ structure of $\mathrm{Al}_{3} \mathrm{Zr}$ precipitates appears for long enough annealing times. On the contrary, Al-Sc system appears to be a good candidate to compare simulated precipitation kinetics with experimentally observed ones as experimental data allowing to quantify the homogeneous precipitation of the $\mathrm{L}_{2}$ structure of $\mathrm{Al}_{3} \mathrm{Sc}$ exists.

Novotny and Ardell [6] as well as Marquis et al. [5,44,45] observed the coarsening behavior of different Al-Sc alloys. For the higher supersaturations studied $\left(x_{\mathrm{Sc}}^{0}=0.18\right.$ at. $\left.\%\right)$, the authors concluded that precipitation occurs homogeneously, at least for temperatures not too high $\left(T \lesssim 350^{\circ} \mathrm{C}\right)$. Therefore, precipitation kinetics of the same alloy can be simulated using cluster dynamics. For the three different temperatures studied $\left(T=300,350\right.$, and $\left.400^{\circ} \mathrm{C}\right)$, it appears that the cluster dynamics equation manage to reproduce the variation with time of the mean precipitate radius (Fig. 7): the agreement is really good with Novotny's data and reasonable with Marquis' ones. Notice that the parameters values used in the cluster dynamics were deduced from the atomic diffusion model used in the kinetic Monte Carlo simulations of the short annealing times of the same Al-Sc alloy maintained at the same temperatures. Cluster dynamics, with a single set of parameters, thus reproduces atomic simulations at short times and gives a safe extrapolation thereof to the range of annealing times that can be compared with experimental data. It should be noticed too that cluster dynamics manages to catch the variation with the annealing temperature of the coarsening kinetics: the experimental observed speed up of the cluster growth rate by a factor $\sim 10$ when going from $T=300$ to $400^{\circ} \mathrm{C}$ is well predicted. The good agreement found for the lower temperatures $\left(T=300\right.$ or $\left.350^{\circ} \mathrm{C}\right)$ depreciates a little bit at higher temperatures. This is due to the fact that, experimentally, for a given nominal concentration one 


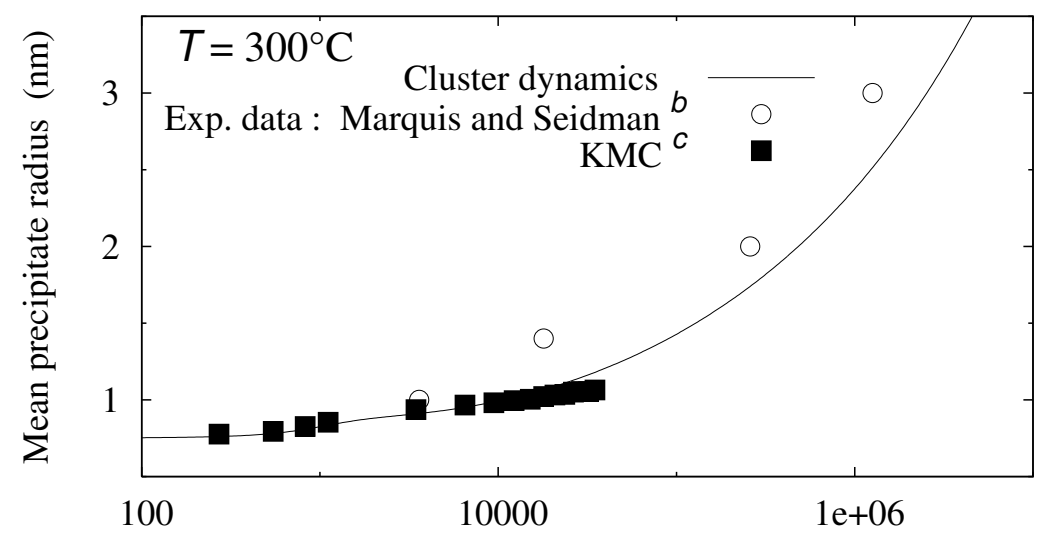

Aging time (s)
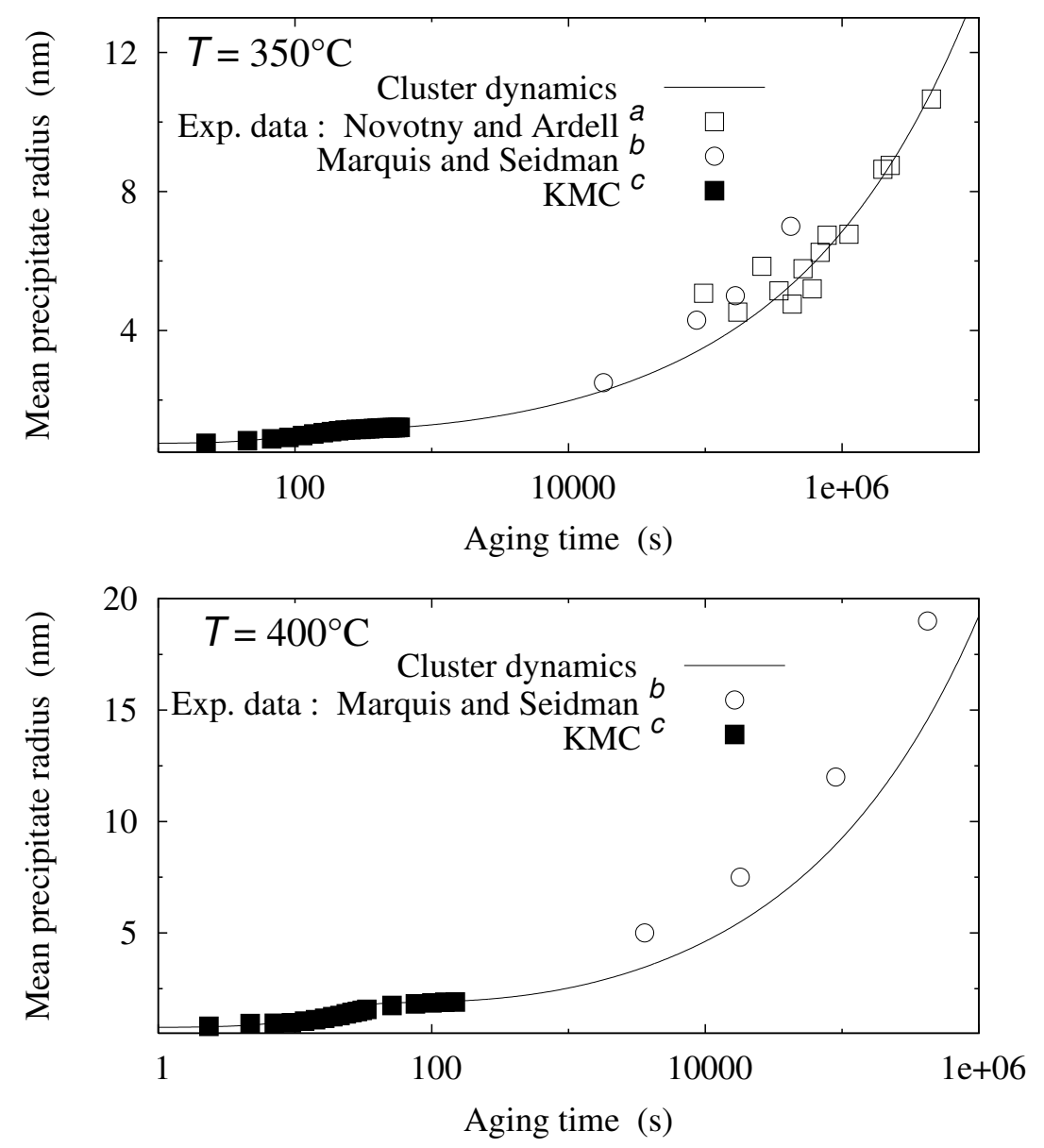

${ }^{a}$ Ref. $6,{ }^{b}$ Ref. 5,44,45, ${ }^{c}$ Ref. 7

Fig. 7. Mean precipitate radius as a function of the aging time for a solid solution of composition $x_{\mathrm{Sc}}^{0}=0.18$ at. $\%$ at different temperature $\left(T=300,350\right.$, and $\left.400^{\circ} \mathrm{C}\right)$ as given by cluster dynamics and compared to experimental data $[5,6,44,45]$ and to kinetic Monte Carlo results [7,8]. The cutoff radius used for cluster dynamics and $\mathrm{KMC}$ is $r_{\mathrm{X}}^{*} \sim 0.75 \mathrm{~nm}\left(n_{\mathrm{X}}^{*}=27\right)$. 
reduces the supersaturation by increasing the temperature and thus favors heterogeneous compared to homogeneous precipitation. There are then less precipitates, these ones being larger.

In figure 8, we compare the precipitate size distribution obtained from cluster dynamics with the experimentally ones measured by Novotny and Ardell [6]. For the different annealing times studied the two normalized distributions are close. Actually, cluster dynamics leads to a normalized size distribution not
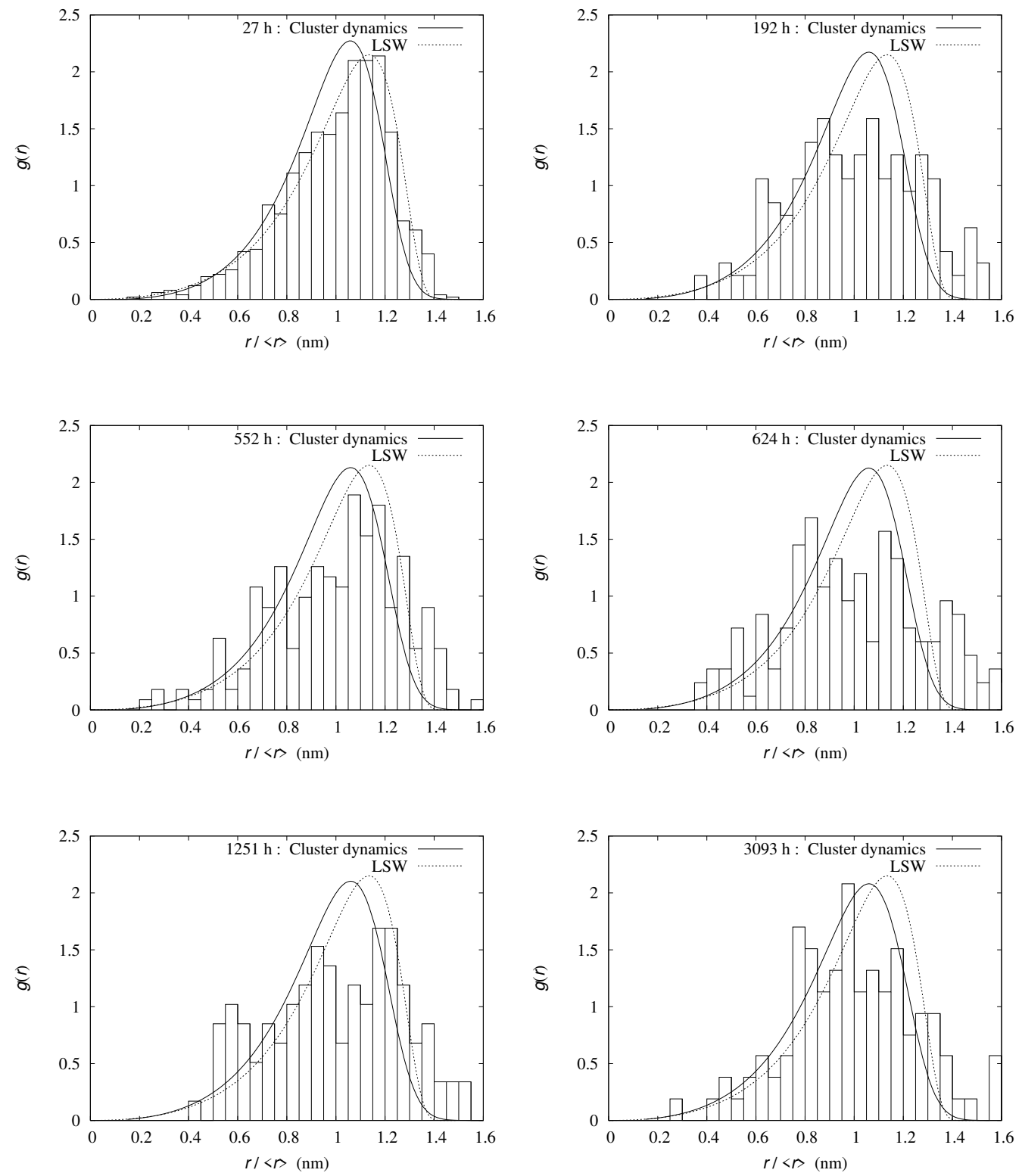

Fig. 8. Normalized precipitate size distributions $g(r)$ for an Al-0.18 at.\%Sc solid solution at $T=350^{\circ} \mathrm{C}$ obtained with cluster dynamics simulations and compared to experimental data [6] as well as to LSW distribution. 
really different from its asymptotic limit, the Lifshitz-Slyozov-Wagner (LSW) distribution. Novotny and Ardell already concluded to a good agreement between experimental size distributions and the LSW distribution, which corresponds to the same agreement found here with the distributions obtained from cluster dynamics.

Hyland [4], using TEM, measured the variation with time of the precipitate density for two different annealing temperatures in an alloy containing 0.11 at.\% Sc. It was not possible to reproduce satisfactorily these experimental data with cluster dynamics. Indeed the simulated densities are higher by at least one order of magnitude than the experimental ones. The number of precipitates experimentally observed depends on the spatial resolution of the observation technique. This can be reproduced by imposing in the simulations a cutoff radius below which the clusters are ignored, even though they might be supercritical. The densities calculated from the simulations are really sensitive to the cutoff radius used to count precipitates. It is possible to obtain lower densities by increasing this cutoff radius but, then, the time scale of the simulations completely differs from the experimental one. The origin of this discrepancy remains an open question.

\section{Conclusions}

Precipitation kinetics of $\mathrm{Al}_{3} \mathrm{Zr}$ and $\mathrm{Al}_{3} \mathrm{Sc}$ in aluminum supersaturated solid solution has been modeled using the cluster dynamics technique. The only input parameters are the solute diffusion coefficients and the precipitate / solid

solution interface free energies. In contrast with other mesoscopic modeling techniques based on Kampmann and Wagner approach, cluster dynamics in its strict sense does not require a definition of the nucleation free energy: this is not an input parameter of the model but rather a by-product of the cluster gas thermodynamic underlying the cluster dynamics equations. We showed that this description leads to a stationary cluster size distribution in the metastable solid solution corresponding to the one predicted by the classical nucleation theory provided CVM is used to calculate the nucleation free energy, in agreement with previous study.

Cluster dynamics is shown to be very sensitive to the interface free energy: the size dependence of the interface free energy, which is revealed by the direct computation of the free energy of small clusters, is crucial in making the cluster dynamics calculations reproduce kinetic Monte Carlo simulations based on the same atomistic parameters. When doing so, the precipitation kinetics obtained agree well with kinetic Monte Carlo simulations: the variations of the precipitate density and of their mean size are well reproduced. Nevertheless, for high supersaturations, kinetics modeled by cluster dynamics appear to 
be slightly too slow. We showed that this discrepancy cannot be solved by an improved kinetic model taking into account the overlap of the cluster diffusion fields.

The cluster dynamics technique as described manages to well reproduce experimental data, despite the fact that the time scales in real experiments are several order of magnitude larger than those in kinetic Monte Carlo. The present study therefore validates, in a quantitative manner, the proposed multiscale scheme for homogeneous precipitation kinetics: atomic scale parameters are obtained from available experimental quantities and ab-initio calculations; mesoscale parameters are deduced from the latter. Cluster dynamics as used in this work, i.e. in its strict sense, then appears as the good tool to extrapolate kinetic Monte Carlo simulations, which reveal full details of the nucleation process, to a wider range of annealing times, of temperatures and of supersaturations. It provides an effective method to accomplish quantitative transition of scales, at least in the low solubility systems which have been considered in this work.

\section{A Equilibrium cluster size distribution}

In cluster dynamics, the size distribution of the clusters in a solid solution at equilibrium is used to deduce the monomer evaporation rates from the condensation rates. This distribution is needed by classical nucleation theory too so as to estimate the cluster size distribution in the metastable solid solution and to deduce from it the steady-state nucleation rate. In the following, we show how this distribution can be obtained for an undersaturated solid solution, as needed by cluster dynamics, the classical nucleation theory extending the validity of this distribution to supersaturated solid solutions.

The cluster size distribution in a solid solution at equilibrium, can be established quite easily as long as the solid solution is dilute [47, 48]. Considering an assembly composed of $N_{n_{\mathrm{X}}}$ clusters containing $n_{\mathrm{X}}$ solute atoms and of free energy $G_{n_{\mathrm{X}}}$, the total free energy of the system can be written

$$
N_{s} G=\sum_{n_{\mathrm{X}}=1}^{\infty} N_{n_{\mathrm{X}}} G_{n_{\mathrm{X}}}+k T \log (\mathcal{W})
$$

where $N_{s}$ is the number of sites that can be occupied by a cluster and $\mathcal{W}$ is the number of different configurations accessible to the cluster assembly. Assuming that each cluster whatever its size lies only on one site and neglecting around each cluster all excluded sites which cannot be occupied by any other cluster, 
this number is simply given by

$$
\mathcal{W}=\frac{N_{s} !}{\left(N_{s}-\sum_{n_{\mathrm{X}}=1}^{\infty} N_{n_{\mathrm{X}}}\right) ! \prod_{n_{\mathrm{X}}=1}^{\infty} N_{n_{\mathrm{X}}} !} .
$$

The concentration of each size class being $C_{n_{\mathrm{X}}}=N_{n_{\mathrm{X}}} / N_{s}$, Stirling formula leads to the following estimation for the total free energy,

$$
\begin{aligned}
G= & \sum_{n_{\mathrm{X}}=1}^{\infty} C_{n_{\mathrm{X}}} G_{n_{\mathrm{X}}}+k T \sum_{n_{\mathrm{X}}=1}^{\infty} C_{n_{\mathrm{X}}} \log \left(C_{n_{\mathrm{X}}}\right) \\
& +k T\left(1-\sum_{n_{\mathrm{X}}=1}^{\infty} C_{n_{\mathrm{X}}}\right) \log \left(1-\sum_{n_{\mathrm{X}}=1}^{\infty} C_{n_{\mathrm{X}}}\right) .
\end{aligned}
$$

The equilibrium concentrations $\bar{C}_{n_{\mathrm{X}}}$ are obtained by minimizing this free energy while imposing that the solute concentration equals a fixed value $x_{\mathrm{X}}^{0}$ :

$$
\sum_{n_{\mathrm{X}}=1}^{\infty} n_{\mathrm{X}} C_{n_{\mathrm{X}}}=x_{\mathrm{X}}^{0}
$$

This can be done quite easily by considering the grand canonic free energy

$$
\mathcal{A}=G+\left(1-2 \sum_{n_{\mathrm{X}}=1}^{\infty} n_{\mathrm{X}} C_{n_{\mathrm{X}}}\right) \mu,
$$

where we have introduced a Lagrange multiplier $\mu$ to ensure the constraint A.4. $\mu=\left(\mu_{\mathrm{X}}-\mu_{\mathrm{A}}\right) / 2$ is nothing else than the effective chemical potential, i.e. half the difference between the chemical potentials of the solvent $\mathrm{A}$ and the solute B. The minimization of this grand canonic free energy leads to the following solution for the cluster size distribution

$$
\frac{\bar{C}_{n_{\mathrm{X}}}}{1-\sum_{j=1}^{\infty} \bar{C}_{j}}=\exp \left(-\frac{G_{n_{\mathrm{X}}}-2 n_{\mathrm{X}} \mu}{k T}\right)
$$

$\Delta G_{n_{\mathrm{X}}}=G_{n_{\mathrm{X}}}-2 n_{\mathrm{X}} \mu$ is the cluster formation free energy relative to the solid solution. It can be estimated using the capillary approximation. As we made the assumption of a dilute solid solution to estimate the total free energy $G$, the sum appearing in the left hand side of equation A.6 can be neglected, so the equilibrium cluster size distribution is simply given by

$$
\bar{C}_{n_{\mathrm{X}}}=\exp \left(-\Delta G_{n_{\mathrm{X}}} / k T\right) .
$$

\section{B Condensation rate}

Monomer condensation rates on clusters are the main input of cluster dynamics. Assuming that the precipitation kinetics is controlled by the solute long 
range diffusion, the condensation rates are obtained by solving the diffusion equation for the solute in the solvent matrix to a spherical cluster with the following boundary conditions: the solute concentration is zero at the cluster (in order to describe solute trapping at the cluster) and far from this cluster the monomer concentration is equal to that corresponding to cluster dynamics simulation. Two cases can be distinguished, depending on whether the cluster is assumed to be isolated or embedded in an effective medium which simulates the trapping to other clusters, i.e. the effect of the overlap of diffusion fields.

\section{B.1 Isolated precipitate}

The rate at which monomers condense on an isolated cluster of radius $r_{n_{\mathrm{X}}}$ is obtained by solving the stationary diffusion equation

$$
D_{\mathrm{X}} \Delta C_{1}(r)=0
$$

with the following boundary conditions: $C_{1}\left(r_{n_{\mathrm{X}}}\right)=0$ at the cluster-matrix interface $^{5}$ and $C_{1}(r \rightarrow \infty)=C_{1}^{\infty}$ far away from the cluster. The monomer concentration profile around the precipitate is thus

$$
C_{1}(r)=C_{1}^{\infty} \frac{r-r_{n_{\mathrm{X}}}}{r}
$$

The condensation rate is given by the integral over the cluster surface of the incoming flux of monomers,

$$
\begin{aligned}
\beta_{n_{\mathrm{X}}} & =-\left.4 \pi r_{n_{\mathrm{X}}}{ }^{2} \frac{D_{\mathrm{X}}}{\Omega} \frac{\partial C_{1}(r)}{\partial r}\right|_{r=r_{n_{\mathrm{X}}}} \\
& =4 \pi \frac{D_{\mathrm{X}}}{\Omega} r_{n_{\mathrm{X}}} C_{1}^{\infty}
\end{aligned}
$$

where $\Omega$ is the mean atomic volume corresponding to one lattice site.

\section{B.2 Precipitate in an absorbing medium}

In order to account for the overlap of the diffusion fields around the many clusters in the matrix, one can compute the diffusion flux to one specific cluster embedded in an effective absorbing medium. The latter extends from infinity

5 Actually, at the interface between the cluster and the matrix, the monomer concentration is equal to the equilibrium one corrected from Gibbs-Thomson effect. Taking this concentration equal to 0 is equivalent to assume that the cluster does not emit any monomer and allows one to directly obtain the condensation rate. 
to a distance $r^{e x t}$ of the cluster, equal to the mean inter-cluster distance. This effective medium is characterized by a constant $k$ giving the rate at which monomers are absorbed, $D_{\mathrm{X}} k^{2}\left(C_{1}(r)-C_{1}^{e q}\right)$. Therefore, for precipitates of radius smaller than $r^{e x t}$, the condensation rate is obtained by solving the set of equations

$$
\begin{aligned}
D_{\mathrm{X}} \Delta C_{1}(r) & =0, \quad r \leq r^{e x t} \\
D_{\mathrm{X}} \Delta C_{1}(r)-D_{\mathrm{X}} k^{2}\left(C_{1}(r)-C_{1}^{e q}\right) & =0, \quad r \geq r^{e x t} .
\end{aligned}
$$

The same boundary conditions apply as previously on the monomer concentration with the additional condition that the diffusion flux has to be continuous at $r=r^{e x t}$. The resolution of this diffusion problem gives the following condensation rate,

$$
\beta_{n_{\mathrm{X}}}=4 \pi \frac{D_{\mathrm{X}}}{\Omega} r_{n_{\mathrm{X}}} C_{1}^{\infty} \frac{1+k r^{e x t}}{1+k\left(r^{e x t}-r_{n_{\mathrm{X}}}\right)} .
$$

For precipitates of size $r_{n}>r^{e x t}$ one has simply to solve the equation

$$
D_{\mathrm{X}} \Delta C_{1}(r)-D_{\mathrm{X}} k^{2}\left(C_{1}(r)-C_{1}^{e q}\right)=0,
$$

still with the boundary conditions $C_{1}\left(r_{n_{\mathrm{X}}}\right)=0$ and $C_{1}(r \rightarrow \infty)=C_{1}^{\infty}$. One obtains for the condensation rate

$$
\beta_{n_{\mathrm{X}}}=4 \pi \frac{D_{\mathrm{X}}}{\Omega} r_{n_{\mathrm{X}}} C_{1}^{\infty}\left(1+k r_{n_{\mathrm{X}}}\right) .
$$

\section{Acknowledgements}

The authors are grateful to Prof. A. J. Ardell and Prof. D. N. Seidman for providing experimental data. They thank too Dr. J.-L. Bocquet, Pr. Y. Bréchet, Dr. J. Dalla Torre, Pr. P. Guyot, Dr. B. Legrand, Dr. J. Lépinoux, Dr. M. Nastar, Dr. C. Sigli and Dr. F. Soisson for fruitful discussions as well as Dr. M. J. Stowell for his careful reading of the manuscript. Part of this work has been funded by the joint research program "Precipitation" between Alcan, Arcelor, CNRS, and CEA.

\section{References}

[1] J. D. Robson, P. B. Prangnell, Dispersoid precipitation and process modelling in zirconium containing commercial aluminium alloys, Acta Mater. 49 (2001) 599-613. 
[2] N. Ryum, Precipitation and recrystallization in an Al-0.5 wt.\% Zr alloy, Acta Metall. 17 (1969) 269-278.

[3] E. Nes, Precipitation of the metastable cubic $\mathrm{Al}_{3} \mathrm{Zr}$-phase in subperitectic Al-Zr alloys, Acta Metall. 20 (1972) 499-506.

[4] R. W. Hyland, Homogeneous nucleation kinetics of $\mathrm{Al}_{3} \mathrm{Sc}$ in a dilute Al-Sc alloy, Metall. Trans. A 23 (1992) 1947-1955.

[5] E. A. Marquis, D. N. Seidman, Nanoscale structural evolution of $\mathrm{Al}_{3} \mathrm{Sc}$ precipitates in Al-Sc alloys, Acta Mater. 49 (2001) 1909-1919.

[6] G. M. Novotny, A. J. Ardell, Precipitation of $\mathrm{Al}_{3} \mathrm{Sc}$ in binary Al-Sc alloys, Mater. Sci. Eng. A318 (2001) 144-154.

[7] E. Clouet, M. Nastar, C. Sigli, Nucleation of $\mathrm{Al}_{3} \mathrm{Zr}$ and $\mathrm{Al}_{3} \mathrm{Sc}$ in aluminum alloys: from kinetic Monte Carlo simulations to classical theory, Phys. Rev. B 69 (2004) 064109.

[8] E. Clouet, Séparation de phase dans les alliages Al-Zr-Sc: du saut des atomes à la croissance de précipités ordonnés, Thèse de doctorat, École Centrale Paris, http://tel.ccsd.cnrs.fr/documents/archives0/00/00/59/74 (2004).

[9] S. I. Golubov, Y. N. Osetsky, A. Serra, A. V. Barashev, The evolution of copper precipitates in binary $\mathrm{Fe}-\mathrm{Cu}$ alloys during ageing and irradiation, J. Nucl. Mater. 226 (1995) 252-255.

[10] M. H. Mathon, A. Barbu, F. Dunstetter, F. Maury, N. Lorenzelli, C. H. de Novion, Experimental study and modelling of copper precipitation under irradiation in dilute FeCu alloys, J. Nucl. Mater. 245 (1997) 224-237.

[11] A. V. Barashev, S. I. Golubov, D. J. Bacon, P. E. J. Flewitt, T. A. Lewis, Copper precipitation in $\mathrm{Fe}-\mathrm{Cu}$ alloys under electron and neutron irradiation, Acta Mater. 52 (2004) 877-886.

[12] L. Lae, P. Guyot, C. Sigli, Cluster dynamics in AlZr and AlSc alloys, in: J. F. Nie, A. J. Morton, B. C. Muddle (Eds.), Proceedings of the $9^{\text {th }}$ International Conference on Aluminium Alloys, Institute of Materials Engineering Australasia Ltd, 2004, pp. 281-286.

[13] G. Martin, Classical nucleation theory and cluster dynamics: the missing link to be published.

[14] A. Hardouin Duparc, C. Moingeon, N. Smetniansky-de-Grande, A. Barbu, Microstructure modelling of ferritic alloys under high flux $1 \mathrm{MeV}$ electron irradiations, J. Nucl. Mater. 302 (2002) 143-155.

[15] T. R. Waite, General theory of bimolecular reaction rates in solids and liquids, J. Chem. Phys. 28 (1958) 103-106.

[16] G. Martin, The theories of unmixing kinetics of solid solutions, in: Solid State Phase Transformation in Metals and Alloys, Les Éditions de Physique, Orsay, France, 1978, pp. 337-406. 
[17] M. Nastar, E. Clouet, Mean field theories for the description of diffusion and phase transformations controlled by diffusion, Phys. Chem. Chem. Phys. 6 (2004) 3611-3619.

[18] J. L. Katz, H. Wiedersich, Nucleation theory without Maxwell demons, J. Colloid and Interface Science 61 (1977) 351-355.

[19] J. S. Langer, A. J. Schwartz, Kinetics of nucleation in near-critical fluids, Phys. Rev. A 21 (1980) 948-958.

[20] R. Wagner, R. Kampmann, Homogeneous second phase precipitation, in: R. W. Cahn, P. Haasen, E. J. Kramer (Eds.), Materials Science and Technology, a Comprehensive Treatment, Vol. 5, VCH, Weinheim, 1991, Ch. 4, pp. 213-303.

[21] A. Deschamps, Y. Bréchet, Influence of predeformation and ageing of an Al-Zn$\mathrm{Mg}$ alloy - ii. modeling of precipitation kinetics and yield stress, Acta Mater. 47 (1) (1999) 293-305.

[22] J. D. Robson, M. J. Jones, P. B. Prangnell, Extension of the N-model to predict competing homogeneous and heterogeneous precipitation in Al-Sc alloys, Acta Mater. 51 (2003) 1453-1468.

[23] R. Kikuchi, A theory of cooperative phenomena, Phys. Rev. 81 (6) (1951) 9881003.

[24] J. M. Sanchez, D. de Fontaine, The fcc Ising model in the cluster variation approximation, Phys. Rev. B 17 (7) (1978) 2926-2936.

[25] H. Bakker, H. P. Bonzel, C. M. Bruff, M. A. Dayananda, W. Gust, J. Horvth, I. Kaur, G. Kidson, A. D. LeClaire, H. Mehrer, G. Murch, G. Neumann, N. Stolica, N. A. Stolwijk, Diffusion in solid metals and alloys, in: H. Mehrer (Ed.), Landolt-Börnstein, New Series, Group III, Vol. 26, Springer-Verlag, Berlin, 1990.

[26] T. Marumo, S. Fujikawa, K. Hirano, Diffusion of zirconium in aluminum, Keikinzoku - J. Jpn. Inst. Light Met. 23 (1973) 17.

[27] S. I. Fujikawa, Impurity diffusion of scandium in aluminum, Defect Diff. Forum 143-147 (1997) 115-120.

[28] S. I. Golubov, A. Serra, Y. N. Osetsky, A. V. Barashev, On the validity of the cluster model to describe the evolution of $\mathrm{Cu}$ precipitates in $\mathrm{Fe}-\mathrm{Cu}$ alloys, J. Nucl. Mater. 277 (2000) 113-115.

[29] A. Perini, G. Jacucci, G. Martin, Cluster free energy in the simple-cubic Ising model, Phys. Rev. B 29 (1984) 2689-2697.

[30] R. C. Tolman, The effect of droplet size on surface tension, J. Chem. Phys. 17 (1949) 333-337.

[31] F. P. Buff, Spherical interface. II. Molecular theory, J. Chem. Phys. 23 (1955) 419. 
[32] J. Lépinoux, Private communication (2004).

[33] I. V. Markov, Crystal Growth for Beginners, World Scientific, Singapore, 1995.

[34] A. D. Brailsford, R. Bullough, M. R. Hayns, Point defect sink strengths and void-swelling, J. Nucl. Mater. 60 (1976) 246-256.

[35] N. Smetniansky-De-Grande, A. Barbu, Study of Cu precipitation mechanisms in $\mathrm{Fe}-\mathrm{Cu} 1.34 \%$ at. alloy under electron irradiation, Radiat. Eff. Defects Solids 132 (1994) 157-167.

[36] N. Smetniansky-De-Grande, A. Barbu, Study of $\mathrm{Cu}$ precipitation in Fe-Cu binary alloys under irradiation. part II, Radiat. Eff. Defects Solids 140 (1997) $313-321$.

[37] O. Izumi, D. Oelschlägel, On the decomposition of a highly supersaturated AlZr solid solution, Scripta Metall. 3 (1969) 619-621.

[38] O. Izumi, D. Oelschlägel, Structural investigation of precipitation in an aluminium alloy containing 1.1 weight per cent zirconium, Z. Metalkd. 60 (1969) $845-851$.

[39] M. S. Zedalis, M. E. Fine, Precipitation and Ostwald ripening in dilute Albased-Zr-V alloys, Metall. Trans. A 17 (1986) 2187-2198.

[40] K. S. Vecchio, D. B. Williams, Convergent beam electron diffraction study of $\mathrm{Al}_{3} \mathrm{Zr}$ in Al-Zr and Al-Li-Zr alloys, Acta Metall. 35 (1987) 2959-2970.

[41] M. E. Drits, J. Dutkiewicz, L. S. Toropova, J. Salawa, The effect of solution treatment on the ageing processes of Al-Sc alloys, Crystal Res. Technol. 19 (1984) 1325-1330.

[42] N. Sano, Y. Hasegawa, K. Hono, H. Jo, K. Hirano, H. W. Pickering, T. Sakurai, Precipitation process of Al-Sc alloys, J. Phys. (Paris) C6 (1987) 337-342.

[43] H.-H. Jo, S.-I. Fujikawa, Kinetics of precipitation in Al-Sc alloys and low temperature solid solubility of scandium in aluminium studied by electrical resistivity measurements, Mater. Sci. Eng. A171 (1993) 151-161.

[44] E. A. Marquis, D. N. Seidman, D. C. Dunand, Creep of precipitationstrengthened $\mathrm{Al}(\mathrm{Sc})$ alloys, in: R. S. Mishra, J. C. Earthman, S. V. Raj (Eds.), Creep Deformation: Fundamentals and Applications, TMS, 2002, p. 299.

[45] E. A. Marquis, Microstructural evolution and strengthening mechanism in Al-Sc and Al-Mg-Sc alloys, Ph.D. thesis, Northwestern University, Evanston, Illinois (2002).

[46] D. N. Seidman, E. A. Marquis, D. C. Dunand, Precipitation strengthening at ambient and elevated temperatures of heat-treatable $\mathrm{Al}(\mathrm{Sc})$ alloys, Acta Mater. 50 (2002) 4021-4035.

[47] J. Frenkel, Kinetic Theory of Liquids, Dover Publications, New York, 1955.

[48] D. Kashchiev, Nucleation : basic theory with applications, Butterworth Heinemann, Oxford, 2000. 